\title{
Evidence Base of Stroke and Hand Splinting
}

\author{
Prabhjot Kaur Gambhir \\ Max Super Specialty Hospital, Saket, New Delhi
}

\begin{abstract}
Post-stroke spasticity results in affected upper extremity posture which hinders daily activities and affects overall quality of life. Among the various rehabilitation options for management of spastic hand, orthosis have been widely used. Literature lacks specific guidelines for type of orthosis, wearing schedule and duration, and material use. Recent researches have emphasized that static splint use has no potential benefit in either reducing muscle tone or providing adequate stretch to shortened muscles and also adds to the discomfort of wearing the splint. Dynamic splinting prevents learned non-use as well as allows patient to use hand during functional activities while maintaining muscle length when no activity occurs. This necessitates the need for evidence based practice for splint use in stroke patients instead of splint prescriptions given by occupational therapists based on preference.
\end{abstract}

Keywords: Stroke, Dynamic splinting, Spasticity, Hemiplegic hand, Functional splinting

\section{Introduction}

Spasticity was first described by Lance in 1980 as a motor disorder characterized by a velocity dependent increase in tonic stretch reflex (muscle tone), with exaggerated tendon jerks, resulting from hyper excitability of the stretch reflex, as one component of the upper motor neuron syndrome'. In 1994, Young added neurophysiological elements to define spasticity.

A study by Wissel et.al showed that $25 \%$ of patients with stroke suffer from spasticity within the first 6 weeks of the event (1). In the upper limbs, the most frequent pattern of spasticity is internal rotation and adduction of shoulder coupled with flexion at the elbow, the wrist and the fingers. Alongside current spasticity preventions such as stretch, exercise, and positioning, treatment options include oral anti-spasm drugs, blocking by phenol, baclofen, and botulinum toxin injections. One strategy for the management of abnormal limb posture resulting from both spasticity and weakness is the use of orthotic devices (2).

An orthosis is defined by the International Standards Organization as an externally applied device used to modify the structural or functional characteristics of the neuromusculoskeletal system (Ponton 1997). An orthosis has two main functions: biomechanical (controlling position), and neurophysiological (reducing tone). The use of splints after stroke can be traced as far back as 1911 (Neuhaus et.al, 1981) (3).

There is controversy about the effectiveness of orthosis, and whether they should be used at all. If they are used, it is controversial at which stage of the natural history of the condition they should be used, the length of time the orthosis should be worn, and within the individual categories of devices, which of the various designs is best employed (3). To make a good splint, therapists must have a good understanding of thermoplastic material properties that include drapability, elasticity, durability, rigidity, flexibility, bonding, and memory. In the acute stroke patient, the immediate goals should be to diminish adaptive muscle shortening, reduce and prevent edema, and protect the joint system (2).
Splinting patients with upper extremity problems continues to remain an enigma in upper extremity rehabilitation. Even among proponents of splinting, numerous disagreements exist concerning splint design, inelastic versus elastic mobilization methods, surface of splint application, joints to be splinted, specific construction materials for splints and splint components, and wearing times and schedules. Problems with studies reported in the literature include weak or limited research design, extremely small population samples, invalid and unreliable methods of measuring spasticity, lack of variable control, lack of consistent methodology.

Traditionally, splints from a wide range of categories have been used in the management of upper extremity, including inhibitory casting, inflatable pressure splints (air splints), custom fabricated low temperature thermoplastic splints, prefabricated or preformed splints, etc. Materials used in the fabrication of these splints include, but are not limited to, low- or high- temperature thermoplastics, plaster, fibreglass, flexible plastic (air splints), neoprene, leather, velcro strapping, cloth towelling, foam, lycra, elastic gloves, and wooden dowels.

Splinting for post stroke spasticity and contractures is contentious and debate continues due to insufficient evidence documenting its effectiveness. Also, inadequate emphasis has been placed on various potential confounders such as lack of rationalization for the time of initiation of splinting treatment, splint wearing schedule, and type/ position of the splint used. Consequently, in the absence of more scientifically validated evaluations, much is left to subjective clinical judgement, resulting in varying splinting practices. This warrants an increased urgency to assess splinting efficacy as well as the variety of splints available for better potential benefits from splinting.

\section{Literature Survey}

Of all stroke survivors, more than half experience impairments of the upper limb in the chronic phase, including loss of strength and dexterity, spasticity, muscle contracture, pain, and edema the abnormal position of the hemiplegic hand and wrist may interfere with daily activities and hygiene maintenance, both negatively influencing the 


\section{International Journal of Science and Research (IJSR) \\ ISSN (Online): 2319-7064}

Index Copernicus Value (2013): 6.14 | Impact Factor (2014): 5.611

quality of life. There are two main rationales which guide clinicians' clinical reasoning for the use of upper limb orthotics: biomechanical to prevent and manage length associated changes in muscles and connective tissue, and neurophysiological approach to inhibit abnormal reflex activity, including spasticity and hypertonia.

The efficacy of hand splinting after stroke has been examined in several reviews. In a cross-sectional survey conducted by Adrienne C et.al in Ireland in 2011 (4), a questionnaire was designed to study the therapists' splint prescription pattern and perception of splint efficacy. While a few occupational therapists chose to correct their splints to accommodate clients' needs through serial static splinting, most preferred volar-based wrist-hand splint as two different splints namely, resting-hand splint (placing the wrist in neutral position and thumb in radial abduction) and functional- position splint (placing the wrist in upto 30 degrees of extension and thumb in opposition).

With regard to splint wearing regimen, there was almost an equal preference for day- splinting and night-splinting. However, there was a mixture of preferences among the therapists for the daily duration of splint use. Therapists believed that through daytime splinting, patients' splint adherence was better achieved due to enhanced comfort. Reducing muscle spasticity, preventing soft tissue contractures, maintaining joint alignment and increasing range of motion were some of the most common reasons for splint prescription. The lack of splinting guidelines seems to be playing a role not only in making decisions about splint prescription based on preference but also in establishing a wearing regimen.

The common parameters employed for assessing splinting efficacy have been found to be range of motion (active or passive), muscle tone, and upper extremity function. Range of motion is measured using standard goniometry, muscle tone with modified ashworth scale and tardieu scale, and upper extremity function using Fugl-Meyer upper extremity assessment, box and block test, functional reach test, and action research arm test $(5,6,7,8,9,10)$. Also, the effect of splinting on ADL components has been studied using COPM (11).

\section{Discussion}

\section{Types of splints}

Lannin et.al (2007) studied the effect of splinting the hand with neutral or extended wrist versus no splinting in a randomized controlled trial of 63 subjects using torque angle, where no difference was found among the groups. They concluded that the routine practice of hand splinting to prevent muscle contracture during acute rehabilitation after stroke should be discontinued (12).

Also, a pre-post comparison of splinting with wrist extension of 30 degrees was done by Amini et.al (2014) (6) to determine the degree the stretch in the splint. A significant amount of reduction in spasticity was measured over a period of 1 month. Although this study refutes the earlier study but is limited by lack of control group and small sample size (14 subjects).
Another RCT by Basaran A et.al (2012) (13) compared dorsal-based, volar-based, and no splinting where no statistically significant difference was found among the groups (39 subjects). Long-term effects of these splints need to be studied to support the conclusions of the same.

\section{Static to Dynamic Trend}

Static splint designs have been used by therapists worldwide for hand and wrist to decrease spasticity, prevent or reduce contractures, improve activity at a joint, protect joint integrity, and reduce pain. However, in a descriptive study conducted by Aukje et.al (2013) (14), they observed that, when used in clinical practice, a considerable amount of stroke patients complain about increased pain and spasticity since the use of static orthosis. Due to discomfort, the orthoses cannot be worn for the advised 8 hours per day which leads to non use in chronic stroke patient and with that increases the risk of developing clenched fists with which patients may experience problems with daily activities and hygiene maintenance. Also, the level of spasticity varies during daytime resulting in different positions of the wrist. The chosen position of the static orthosis is seldom adequate to manage these varying levels of spasticity and changing ranges of wrist mobility.

They also studied the tolerance effect of a dynamic wrist hand orthosis on the prevention of spasticity and progressive wrist contracture after stroke (2013) (15). The results indicated increased tolerance for the dynamic orthosis for atleast 6-hours daily with significantly reduced wrist contractures over a 6- month period.

\section{The advent of Saebo-orthosis}

Patients who demonstrate moderate to severe upper extremity hemiparesis are unable to functionally engage their upper limb. Consequently, learned non-use is thought to further decrease movement and increase pain. The inability to effectively incorporate the paretic limb into tasks can lead to decreased cortical activation and long term functional limitations. A recent advancement in the orthotic prescription for stroke patients has been Saebo's Functional Neurological Dynamic Orthosis.

Saeboflex is a dyanamic custom-fabricated wrist, hand, finger orthosis (WHFO). The forearm shell and hand piece are fabricated to a specific patient's clinically derived measurements and the orthosis includes a dynamic finger and thumb extension assist. The extension system is comprised of resistive springs of varied force. Four finger lead mounts function like outriggers on other dynamic orthosis. The thumb also has a similar mounting system. Digit caps are used to block the DIP joints of the fingers and the IP joint of the thumb. The optimum wrist angle of the SaeboFlex is positioned at 35 degrees of extension. If the patient has increased tone/spasticity and/or soft tissue shortening, thena 15 degrees of wrist mount if recommended. The primary benefit of the SaeboFlex in this population is the facilitation of massed practice and improvement in motor control and overall arm function (7, $8,10)$. 


\section{International Journal of Science and Research (IJSR) \\ ISSN (Online): 2319-7064}

Index Copernicus Value (2013): 6.14 | Impact Factor (2014): 5.611

SaeboReach is a dynamic custom-fabricated elbow, wrist, hand, finger orthosis (EWFHO). The proximal component includes an elbow cuff that assists with extending the elbow during functional reaching. It can be adjusted by the therapist to facilitate or inhibit supination or pronation during grasping.

The SaeboStretch is a dynamic progressive splint. It includes three 'energy storing' hand plates that offer various grades of resistance. Therefore, as tone increases in the patient's hand, the fingers flex in the splint instead of pulling out. The dynamic hand piece then repositions the fingers into extension as tone subsides.

The effectiveness of SaeboFlex orthosis was studied by Farrell JF et.al (2007) (8) resulting in an improvement in upper limb mobility and grasp capacity. The orthosis aimed at goal-directed activities with repetitive practice. A comparative pre-post design was used and goniometry, MAS, Fugl-Meyer assessment was used as outcome measures.

A multiple single-case design study was conducted by Franck JA (2013) (A-B-A design) (10). 8 subjects were trained with SaeboFlex orthosis and found to improve on their ability to use their affected arm during and after training on ARAT and ABILHAND (measuring patient's performance on actual real like task performance. However, as the patients were in the subacute phase after stroke, this improvement might have (partly) been due to several factors like spontaneous recovery or the regular rehabilitation that the patients received.

A RCT was done by Hye-Seon Jeon et.al (2012) where 10 subjects showed improvement in finger extension for impaired grip opening with SaeboFlex orthosis. Movement smoothness improved as noted by ARAT (7).

\section{Conclusion}

\section{1) Splinting to decrease spasticity}

- The points of contact of a splint are thought by some clinicians to impact on whether or not a splint inhibits or elicits spasticity. Such thoughts have stemmed from early publications (Rood) where spasticity was thought to increase as a result of sensory stimulation of the palmar surface of the hand, which would then result in unwanted muscle contractions. Based on these assumptions, many therapists recommend splinting on the dorsal surface of the hand only.

- There is strong evidence that wearing a splint all night has no additional effect in reducing spasticity over usual therapy. Furthermore, even wearing the splint upto 22 hours per day did not affect spasticity. Results from studies also suggest that there is no difference between using a dorsal splint to a volar splint. This is not surprising since people after stroke rarely exhibit exaggerated cutaneous reflexes.

\section{2) Splinting to decrease contracture}

- Contracture is the loss of joint range of motion. This is partially the result of a shortening of muscle length due to decrease in the number of sarcomeres in series along with myofibrils. There is strong evidence that wearing hand splints all day and night additional to usual therapy after stroke has no effect in preventing contracture, whether the wrist is splinted in neutral or in maximum extension. Furthermore, it appears that there is no difference between using a splint to other means of contracture prevention.

\section{3) Splinting to improve activity}

- Early and/or continuous splinting predisposes a patient to learn non-use. one of the disadvantages of suing splinting is that it effectively immobilizes the joints and therefore discourages or even disallows muscle activity and therefore movement.

- A major obstacle to functional use of the hand is the inability to open the hand spontaneously, and the potential benefit of dynamic and newer technology splints on hand opening, activity and functional use has yet to be adequately studied in clinical trials.

\section{References}

[1] Thibaut A, Chatelle C, Ziegler E, Bruno MA. Spasticity after stroke: physiology, assessment and treatment. Brain Injury, 2013, Vol 27, Issue 10, pp. 1093-1105. Doi: 10.3109/02699052.2013.804202

[2] Pitts DG, O'Brien SP. Splinting the hand to enhance motor control and brain plasticity. Top Stroke Rehabilitation, Sept-Oct 2008, Vol 15, Issue 5, pp. 456467

[3] Kent RM, Gilbertson L, Geddes JML. Orthotic device for abnormal limb posture after stroke or nonprogressive cerebral causes of spasticity. Cochrane Collaboration,

2008 .

DOI: $10.1002 / 14651858 . C D 003694$

[4] Adrienne C, Manigandan C. Inpatient occupational therapists hand splinting practice for clients with stroke: a cross-sectional survey from Ireland. J Neurosci Rural Pract., 2011, Vol 2, Issue 2, pp.141-149

[5] Shamili A, Amini M, Forough B, Kazemi K, et.al. Botulinum toxin injection or application of splints: impact of spasticity, range of motion, and function of upper extremity in stroke patients. Iranian J Pharmacol and Theraputics, 2011, Vol 10, pp. 11-16

[6] Amini M, Moosavi S, Shamili A, Kazemi R et.al. Impact of extension splint on upper extremity motor components and function in chronic stroke patients. Iranian Rehabil J, 2014, Vol 12, Issue 1, pp. 18-21

[7] Jeon HS, Woo YK, Yi CH, Kwon OY, et.al. Effects of intensive training with a spring-assisted hand orthosis on movement smoothness in upper extremity following stroke: a pilot clinical trial. Top Stroke Rehabil, Jul-Aug 2012. Vol 19, issue 4, pp. 320-328

[8] Farrell JF, Hoffman HB, Snyder JL, Giuliani CA, et.al. Orthotic aided training of the paretic upper limb in chronic stroke: results of a phase I trial. Neurohabil, 2007, vol 22, Issue 2, pp. 99-103

[9] Jo HM, Song JC, Janq SH. Improvements in spasticity and motor function using a static stretching device for people with chronic hemiparesis following stroke. Neurorehabil, 2013, Vol 32, Issue 2, pp. 369-375

[10] Franck J, Timmermans AAA, Seelen HAM. Effects of a dynamic hand orthosis for functional use of the impaired upper limb in subacute stroke patients: a

\section{Volume 5 Issue 2, February 2016}




\section{International Journal of Science and Research (IJSR) \\ ISSN (Online): 2319-7064}

Index Copernicus Value (2013): 6.14 | Impact Factor (2014): 5.611

multiple single-case experimental design study. Technology and Disability, 2013, Vol 25, pp. 177-187

[11] Garros DSC, Gagliardi RJ, Guzzo RAS. Evaluation of performance and personal satisfaction of the patients with spastic hand after using a $\mathrm{v}$ olar-dorsal orthosis. Arch Neuro - Psych, June 2010, vol 68, Issue 3. DOI: 10.1590/S0004-282X2010000300011

[12] Lannin NA, Cusick A, McCluskey A, Herbert RD. Effects of splinting on wrist contracture after stroke: a randomized controlled trial. Stroke, Jan 2007, Vol. 38, pp. 111-116

[13] Basaran A, Emre U, Karandavut KI, Balbaloqlu O, et.al. Hand splinting for post stroke spasticity: a randomized controlled trial. Top Stroke Rehabil, 2012, Vol 19, Issue 4 , pp. 329-337

[14] Andringa A, Port IVD, Meijer JW. Long-term use of a static hand-wrist orthosis in chronic stroke patients: a pilot study. Stroke research and treatment, 2013, DOI: $10.1155 / 2013 / 546093$

[15] Andringa AS, Van de Port IG, Meijer JW. Tolerance and effectiveness of a new dynamic hand-wrist orthosis in chronic stroke patients. Neurorehabil, 2013, Vol 33, Issue 2, pp.225-231

\section{Author Profile}

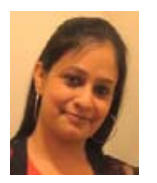

Prabhjot Kaur, MOT (Neurosciences) is an Occupational Therapist at Max Super Speciality Hospital, Saket, Delhi (India). Her field of work involves adult and paediatric neuro- rehabilitation including dysphagia management. She is certified in Vital Stim Therapy and conservative Dysphagia management as well as Breast Cancer rehabilitation. She has received a Service Excellence Award (GEM- Going an Extra Mile) during her work tenure at Max Hospital. Also, she has been on the review board of Education Research Journal. She has international publications in Dysphagia , Neuro- rehabilitation and Breast cancer rehabilitation. 